\title{
Adaptive Multi-channel MAC protocol for dense VANET with Directional Antennas
}

\author{
Xu Xie ${ }^{1}$, Benxiong Huang ${ }^{1}$, Shaoshi Yang ${ }^{2}$ and Tiejun $\mathrm{Lv}^{2}$ \\ ${ }^{1}$ Department of Electronics and Information Engineering, \\ Huazhong University of Science and Technology, Wuhan, China \\ ${ }^{2}$ School of Information and Communication Engineering, \\ Beijing University of Posts and Telecommunications, Beijing, China \\ Contact: xiesky@163.com
}

\begin{abstract}
Directional antennas in Ad hoc networks offer more benefits than the traditional antennas with omni-directional mode. With directional antennas, it can increase the spatial reuse of the wireless channel. A higher gain of directional antennas makes terminals a further transmission range and fewer hops to the destination. This paper presents the design, implementation and simulation results of a multi-channel Medium Access Control (MAC) protocols for dense Vehicular Ad hoc Networks using directional antennas with local beam tables. Numeric results show that our protocol performs better than the existing multichannel protocols in vehicular environment.
\end{abstract}

Keywords: Directional antennas; vehicular ad hoc network; multi-channel; beam table

\section{INTRODUCTION}

Recently, vehicular Ad hoc network (VANET) [1] attracts much attention in wireless communication field. The spectrum following the Federal Communications Commission (FCC) standard Dedicated Short-Range Communications (DSRC) [2] is divided into seven channels for vehicle-to-vehicle, vehicleto-roadside communication and safety service. A main difference between VANET and conventional Ad hoc network is a variable network density caused by the rapid changes of network topology. In highway, the density of vehicles is likely more lower than that in urban. Traffic would become serious during the rush hours, while a sparse vehicles scenario are expected to appear at night or other idle daytime. In a high density environment, a large number of vehicles with wireless transceivers are co-exist in a communication zone [3], and the channel access contention problem is a crucial challenge for reliable transmissions with low delay and an efficient protocol based on DSRC standard. The multi-channel scheme was first proposed in 1983 [4] for throughput improvement of local area network (LAN). But in dense Ad hoc networks, some questions are still to be answered:

1) If the number of nodes is larger than the allocated channels. How to improve the channel capacity?

2) While choosing the common channel, how to avoid repeated collisions?
3) How to achieve a higher throughput with a relatively low end-to-end delay?

\section{RELATED WORK}

Early works are designed for CSMA or ALOHA protocols in slotted multiple channels [5] for wired networks. Latter, $\mathrm{Wu}$ et al. proposed a protocol named Dynamical Control Assignment (DCA) [6] based on the dedicated channel assignment. It assumes that each node equipped with two transceivers, one is used for overhearing the control messages, such as RTS/CTS, the other is used for transmitting data packets. So it can achieve concurrent communication in control channel and data channel. Because of using multiple transceivers simultaneously, the synchronization is not needed here and modifications of protocol would be fewer in each channel. But it is costly utilizing two transceivers.

Nasiquri in [7] proposed a "soft" channel reservation scheme which assumes that each node can listen to all of its channels simultaneously and transmits control/data packets in the idle channels. In its conclusion, reservation based scheme performs better than a multi-channel scheme with random selection of idle channels. But a large number of channels are required in this scheme, and it may cause an unacceptable transmission delay. Another problem of Nasiquri's scheme is the random channel switching which may cost a lot in switching procedure and lead a frequent channel changes.

In [8], Porwal and Papadopouli's method focuses on switching of multiple idle channels based on the traffic conditions of channels and communication pattern of each host. Two messages departure (DEP) and arrival (ARR) are utilized for computing the traffic share of each channel. A threshold of share can decide whether a switch would happen or not. The scheme achieves an explicit power-savings mode and can be based on 802.11 extensions without any complicated additional hardware requirement.

McMAC [9] adopts an approach that a channel reservation period is used to choose a common idle channel for the coming communications between senders and receivers. All equipments meet on a default channel exchange control messages to decide how to assign themselves to the available channels during the data phases compared with control phase. 
In the control phase, a preferable channel list which records the usage of channels inside the transmission range of the node is used for channel negotiation. The nodes are synchronized by beacons. In its conclusion, McMAC performs significantly better than DCA in throughput. But in actual communication scenario, the number of available channels would be limited, and in each channel, only two nodes can exchange messages simultaneously.

\section{PRELIMINARIES}

\section{A. IEEE $802.11 p$}

The current IEEE 802.11p draft is used for the future vehicle-to-vehicle (V2V) and vehicle-to-roadside (V2R) communication, which aims at providing the standard specifications to ensure the interoperability between wireless devices embedded in vehicles in rapidly changing communication environments and in situations where transmission must be done in a shot interval.

Based on IEEE 802.11 standard, 802.11p protocol support the Intelligent Transportation System (ITS) applications, which includes the data exchanges in high speed V2V or V2R environments using the licensed ITS band of $5.9 \mathrm{GHz}$. This $5.9 \mathrm{GHz}$ Dedicated Short Range Communications (DSRC) spectrum is divided into seven $10 \mathrm{MHz}$ channels with $10 \mathrm{MHz}$ channel spacing between the centers of the channels [10]. In figure 1, channel 178 is designated as the Control Channel $(\mathrm{CCH})$ where announcements and short data messages are transmitted. The other channels are designated as Service Channels (SCHs), where additional data transfers and special operations applications. Channel 172 and Channel 184 are dedicated for safety application in $\mathrm{V} 2 \mathrm{~V}$ and intersections environments separately.

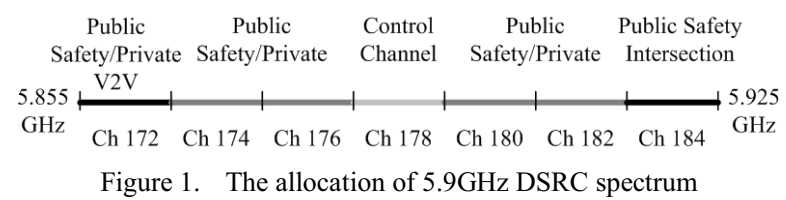

\section{B. Directional Antennas Model}

The antenna model has two separate modes: Omnidirectional and Directional [11]. The omni-directional antennas radiates or receives equally well in all directions. The predefined number of beams should be different with the scenarios. The number that covers the area around the transmitter should be 2 in the highway traffic scenario, compared with 4 in the urban scenario. It is also called the "non-directional" antenna because it does not favor any particular direction.

\section{The Proposed Protocol VMMAC}

The proposed VMMAC (VANET Multi-channel MAC) schemes are derived from McMAC which is designed for Adhoc wireless network. Before describing the protocol in detail, some fundamental assumptions should be summarized.
- Dense vehicular networks allow high instrumented vehicle density, which means that one vehicle would be in many others' communication ranges simultaneously, but incurred plenty of collisions of packets.

- Each vehicle equipped with a single half-duplex transceiver. It means that a vehicle can either transmit or sense, but cannot do both simultaneously. When one listen to one channel, it cannot sense carrier on the other channel. And when one transmits packets in one channel, it cannot interfere in other channels.

- $\quad$ Each vehicle continuously alternates between the $\mathrm{CCH}$ and one of the SCHs or the safety channels. Due to the strong delay requirements of safety application, such as Cooperative Collision Avoidance [12] which limits the delay of a packet transmission in 0.1 second, the switching time is less than 100us [13], which can be ignored in simulation.

- Synchronization should be reached by beacons which are periodically sends in each packet. The beacon includes a timestamp of the local sender's timer. On receiving this beacon, receiver adjusts its local timer to fit the timestamp.

The key point of our scheme is using directional antennas in multi-channel network to achieve the spatial reuse. Follow the antenna model, when using directional antennas, even most of beams are blocked because of having overheard the busy state of channels, the unblocked beams can also communicate with each other as long as the nodes are in unblocked state. With multi-channel technology, although the two preconnecting nodes are in communication range of the senders and receivers, they may set up a wireless link in available unblocked channel. These two characters result in network performance improvement, especially with dense Ad hoc network in vehicular environment.

\section{A. Beam Table}

Before sending the RTS/CTS handshake, there will be a stage to select the communication channel. In [14], an additional stage based on Ad hoc Traffic Indication Messages (ATIM) for a favor channel reservation strategy has been proposed. With channel negotiation during ATIM window, a preferable channel list structure is exchanged. In our scheme, channel selection is contained in RTS/CTS using a beam table (BT), which in local area of each node indicates the current state of beams in all seven DSRC channels. The Table I below provides the BT of node A. Bit ' 1 ' indicate that the beam is blocked in the corresponding channel. It illustrates that beam 1 is blocked state in channel 172 and channel 184, and beam 2 is unblocked in all channels except channel 180 . At the destination, BT of node D is shown in the second table below. Beam 1 in channel 182 and beam 2 in channel 174 are blocked. When node D has received the RTS from node A including its BT, it would conduct a common unblocked $\mathrm{SCH}$ 176 through a comparison between two tables. In addition, if there are more than 1 channel are unblocked in both two BTs, 
the choice should follow the last communication, which can reduce neighbors' switching time, although it is ignored in our simulation.
TABLE I. BEAM TABLE OF NODE A

\begin{tabular}{|c|c|c|c|c|c|c|c|}
\hline $\begin{array}{c}\text { Channel } \\
\text { Beam }\end{array}$ & 172 & 174 & 176 & 178 & 180 & 182 & 184 \\
\hline 1 & 1 & 0 & 0 & 0 & 0 & 0 & 1 \\
\hline 2 & 0 & 0 & 0 & 0 & 1 & 0 & 0 \\
\hline
\end{tabular}

TABLE II. BEAM TABLE OF NODE D

\begin{tabular}{|c|c|c|c|c|c|c|c|}
\hline $\begin{array}{c}\text { Channel } \\
\text { Beam }\end{array}$ & 172 & 174 & 176 & 178 & 180 & 182 & 184 \\
\hline 1 & 0 & 0 & 0 & 0 & 0 & 1 & 0 \\
\hline 2 & 0 & 1 & 0 & 0 & 0 & 0 & 0 \\
\hline
\end{tabular}

\section{B. Directional Handshake}

In directional handshake scheme the RTS/CTS is transmitted directional consecutively, in a certain way. The transmitter starts transmitting a Channel Selection Request To Send (CS-RTS) in a predefined direction, assume with beam 1 . A certain time afterwards it turns its transmission beam on another sending the same CS-RTS. The procedure continues until the transmission of CS-RTS covers all the beams around the transmitter.

The CS-RTS packet contains IDs of transmitter and receiver, beams of their directional antennas, the duration of the intended four way handshake and a BT of the sender. As this information is spread around by the CS-RTS, the neighbors are informed about the intended transmission. The neighbors, after executing a simple algorithm that is described later in this section, decide if they will defer their transmission in the direction of transmitter or receiver, if this harms the ongoing transmission. In this way, the neighbors are aware of the intended handshake, a fact that results in reduction of the hidden terminal problem.

When the CS-RTS finishes transmitting, the sender waits for corresponding Channel Selection Clear To Send (CS-CTS) for the receiver. An omni-direction mode is adopted for sensing the $\mathrm{CCH}$, as 802.11. On the other hand, neighbors of the sender who have overheard the CS-RTS will keep quite and delay the incoming transmission. In destination node, once the CS-RTS is received correctly, there will be a comparison between sender's BT and local BT. A common unblocked SCH would be reserved for the data transmission.

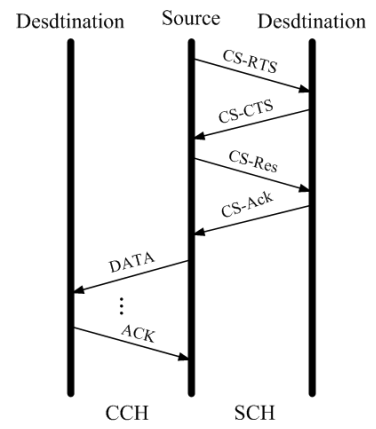

Figure 2. Directional Handshake

Following the sender's beam in CS-RTS packet, the receiver sends back directional CS-CTS using its local receiving beam towards the transmitter. The CS-CTS includes the IDs and beams of the communication pair, the common unblocked channel ID, similar to the CS-RTS. After finishing the transmission, the destination node set his status into waiting for a Channel Selection Reservation (CS-Res) packet.

\section{C. scheme 1: VMMAC with Lane-directional CS-RTS}

VMMAC protocol includes two schemes for directional handshake in $\mathrm{CCH}$. In scheme 1 , a lane-based antenna is utilized for directional transmission, where CS-RTS packets are transmitted in all beams consecutively. As mentioned in Channel Selection stage, CS-RTS packets are sent in a circular way, until it scans all the beams. Figure 2 shows that node A sends a CS-RTS with directional antenna's beam 1 in $\mathrm{CCH}$. A timer in node A starts simultaneously. The transmission has not been changed until the timer ends and no CTS packet has been received.

In directional handshake scheme the RTS/CTS is transmitted directional consecutively, in a certain way. The transmitter starts transmitting a Channel Selection RTS (CSRTS) in a predefined direction, assume with beam 1. A CSRTS transmission time and a short inter-frame space (SIFS) afterwards it turns its transmission beam on another sending the same CS-RTS. So the average time for sending a CS-RTS is:

$$
T_{C S-R T S}=k * T_{R T S-\text { timeout }}+\text { SIFS }
$$

Where $\mathrm{k}$ is the number of beam in which a CS-CTS of destination node has been received. Thus, the minimum of $\mathrm{k}$ is 0 and the maximum is the $\left(n_{\max }-1\right)$, where $n_{\max }$ is the max number of beams, unless the destination node is out of the communication range of the source node. In highway scenario, the number of beams in a vehicle is 2 , and the $\mathrm{T}_{\text {CS-RTS }}$ may be low. However, in urban scenario or other environments with high density of nodes, the number of a node would be an efficiency problem, which should be correlated with classification of directions.

In figure 3 , node $\mathrm{A}$ have a message for node $\mathrm{D}$ and initiate its CS-RTS in CCH using beam 1. Traditionally, when node E has overheard the packet, the node is set blocked state and will not receive other messages until unblocked state comes. If another node who did not know the state of the node E sends a message to it, a typical hidden terminal problem has been exist.

To increase the spatial reuse and reduce the hidden terminal problems, here are two principles for the BT:

a) when a node is idle, its unblocked beams could receive any directional or omni-directional messages;

b) only when a node is idle and all of its beams are blocked, it would change its current SCH into another for communications.

Using BTs, when a node which is not the destination receives the CS-RTS, it just modifies the item of its BT into blocked state. In another period of $\mathrm{CCH}$, it can also have a communication with others. For example, as shown in figure 3 , after 4 directional handshakes, the beam 2 of node $\mathrm{E}$, all beams of node $\mathrm{B}$ and node $\mathrm{C}$ are in blocked state with channel 
172. Follow principle a), if node $\mathrm{F}$ wants to communicate with node $\mathrm{E}$, while sending CS-RTS to node $\mathrm{E}$ in $\mathrm{CCH}$, node $\mathrm{E}$ can be a receiver using unblocked beam 1 in current channel. So as to node $\mathrm{B}$ and node $\mathrm{C}$, all beams of nodes are in blocked in channel 172 because of having overheard the transmissions between A and D. According to principle (b), if node B has a message for node $\mathrm{C}$, they have to negotiate with each other in exchanging BT. The common idle items would be the candidate of the next communication channel.

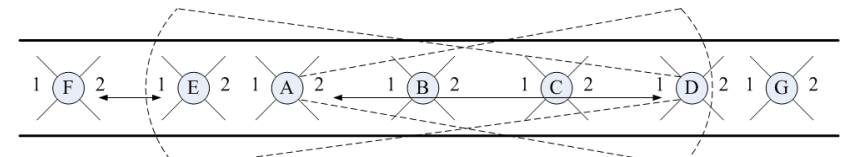

Figure 3. Hidden terminal problem in highway scenario

\section{D. scheme 2: VMMAC with Omni-directional RTS}

Scheme 1 using a lane-based directional antenna is fit for sparse traffic environment with lane limits, such as highway, suburb and etc. In urban scenario, messages would be received in all directions as the heavy traffic makes a high density of vehicles. When more than three nodes exist in one communication zone simultaneously, they would face a contention of accessing the same channel. When the destination node is not in 1-hop range of the source node, a multi-hop scheme should be operated, and the directional RTS would cost a lot in duration of beam switching. Scheme 2 can alleviate the problem by sending an omni-directional RTS, in spite of being reduced the communication distances, a multihop scheme would be a complement.

\section{PERFormance EVALUATION}

To evaluate our protocols, we compare the DCA, McMAC with VMMAC. Simulations are operated in two scenarios, one-lane highway platoon and random way urban environments. According to $802.11 \mathrm{~b}$ based parameters, the omni-directional radio range of each node is $300(\mathrm{~m})$, comparing with the directional radio range $500(\mathrm{~m})$, the CBR is 50 flows with random sources and destinations, and the data packet size is 1 (kbytes). As mentioned in section above, the number of channels is 7 with 1 control channel and 6 service channels. Assume that each channel has the same bit rate 2 (Mbps), and the channel switching time is 100 (us). To study the impact of these factors on the throughput, packet delay and probability of successful transmissions, we performed simulations varying parameters.

Before the simulation, an important characteristic should be considered here, the density of nodes in a radio range. When the distance between adjacent vehicles $i$ and $i+1$ which travel in the same communication zone is less the omnidirectional radio range, both two schemes can be effective. When the distance is more than that but less than the directional radio range, only scheme 1 can be used. Otherwise, a disconnection problem exists. So an analysis of average

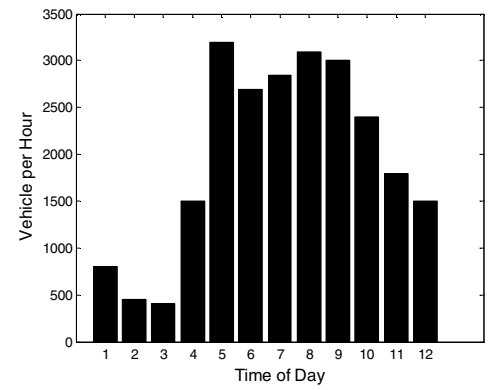

Figure 4. Actual Traffic flow collected by BHL

spacing of neighbors promoted below for a more accurate description of the dense VANET traffic.

\section{A. Average Spacing of Neighbors $\left(E\left[D_{\text {neg }}\right]\right)$}

In dense vehicular networks, packets travel across a number of vehicles by multi- or single-hop communication, where the number of hops within depends on the number of vehicles in a communication zone and the spacing between two neighbor vehicles. Assume that the inter-vehicle spacing $S$ follows an exponential distribution with parameter $\lambda_{\mathrm{S}}$, whose probability distribution function (PDF) can be expressed as:

$$
f\left(d_{n e g}\right)=\operatorname{Pr}[S \mid S \leq R]=\frac{\lambda_{S} e^{-\lambda_{S} d_{\text {neg }}}}{1-e^{-\lambda_{S} R}}
$$

and the average spacing of neighbors is:

$$
E\left[d_{n e g}\right]=\frac{1}{\lambda_{S}}-\frac{R e^{-\lambda_{S} R}}{1-e^{-\lambda_{S} R}}
$$

\section{B. Simulation Results}

An empirical model from the real freeway traffic data we have conducted are collected by the Berkeley Highway Laboratory (BHL) [15]. As shown in figure 4, three different time periods with different traffic flow behaviors were carefully collected with each time period lasting for 2 hours.

For highway scenario, the exponential distribution with the parameter $\lambda_{\mathrm{S}}$ obtained as $0.00625(\mathrm{veh} / \mathrm{m})$ during the busy time. And the average spacing of neighbors here equals 54.33 (m).

As shown in figure 5, the throughput of directional VMMAC with two radios is the best among all MAC protocol schemes. This is because directional antennas with long distance focusing transmission power into one beam remarkably improve the average data rate. With two radios, the bandwidth bottleneck of control channel that has a serious limitation of network capacity in DCA can be alleviated. When using one radio in directional and omni-directional VMMAC, the throughput is little lower than the DCA, which has two transceivers for control channel and data channel respectively. Due to the high density of vehicles, throughput of McMAC without multiple channels corrupts seriously by consecutive collisions of channel contention, although McMAC achieves a better performance than DCA in pure Ad hoc network. 


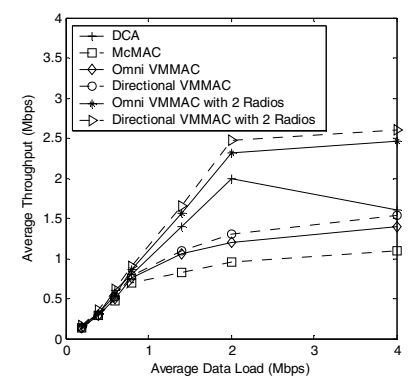

Figure 5. Average throughput of VMMAC schemes Figure 6. in highway scenario

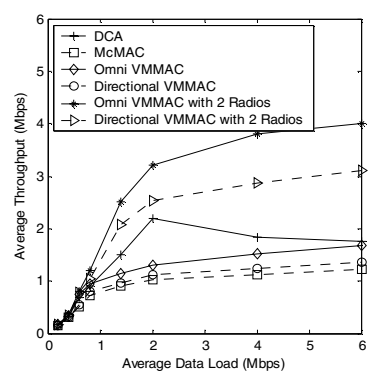

Average throughput of VMMAC schemes in urban scenario

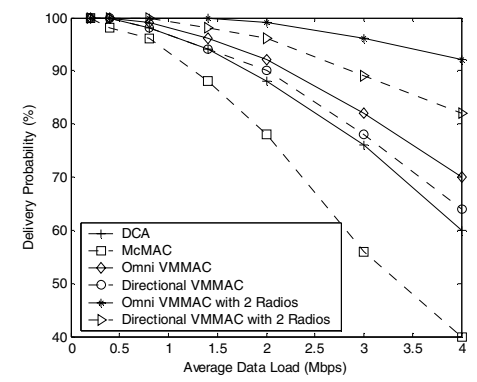

Figure 7. Comparison of delivery probabilities in urban scenario
In urban scenario, 100 nodes are randomly placed in a 1 $(\mathrm{km}) * 1(\mathrm{~km})$ area, in which 30 nodes are randomly chosen to be sources, and 30 nodes are chosen to be destinations. $\lambda_{\mathrm{S}}$ is assumed as $0.00958(\mathrm{veh} / \mathrm{m})$. A node may be the source for multiple destinations, and a node may be the destination for multiple sources. In figure 6 , as expected, VMMAC still perform better than other two protocols, and omni-directional schemes outperform the directional schemes. It can achieve 4.1 (Mbps) using omni-directional VMMAC with two radios, when average data load is 6 (Mbps). But the DCA drops to only 1.6 (Mbps).

Figure 7 shows a comparison of delivery probabilities in urban scenario. At 500 (Kbps) traffic load, the all the protocols have the similar probability close to $100 \%$. As the traffic increases, the probability of McMAC declines more than the rest schemes. Because of the feature that directional antennas can effectively separate the contending transmissions, the omni-directional VMMAC with two radios maintains an up-to $91 \%$ delivery probability.

\section{CONCLUSIONS AND FUTURE WORK}

In this paper, we have presented a multi-channel MAC protocol using directional antennas for dense VANET. The current multi-channel protocols, which are restricted by the number of channels, enable few terminals to access in channels. Using directional antennas, our proposed scheme allows pairs of terminals with idle beams to connect in the same channel. Two schemes are proposed for highway and urban scenarios using lane-directional and omni-directional antennas separately. The simulation result shows that, in the dense nodes environment, our scheme can achieve a better performance in throughput and a higher probability of reliable transmissions. As further study, we will focus on an asynchronous mode in channel selection strategy and a speed adaptive technology for VANET.

\section{REFERENCES}

[1] Q. Xu , T. Mak and J. Ko, "Vehicle-to-vehicle safety messaging in DSRC," Proceedings of the 1st ACM international workshop on Vehicular ad hoc networks, Philadelphia, PA, USA, Oct, 2004.

[2] http://www.dsrc.com/

[3] Briesemeister, L. Schafers and L. Hommel, "Disseminating messages among highly mobile hosts based oninter-vehicle communication,"
Proceedings of Intelligent Vehicles Symposium, MI, USA, pp.522-527, Oct 2000.

[4] A. M. Marsan and D. Roffinella, "Multichannel local area network protocols," IEEE JSAC, vol. SAC-1, no. 5, pp. 885-897, Nov 1983.

[5] A. Nasipuri and S. R. Das, "Multichannel CSMA with signal powerbased channel selection for multihop wireless networks," Proceedings of the IEEE Fall Vehicular Technology Conference, Sep 2000.

[6] S. L. Wu, C. Y. Lin and Y. C. Tseng, "A Dynamic Multi-Channel MAC for Ad-Hoc LAN," Proceedings of International Symposium on Parallel Architectures, Algorithms and Networks (ISPAN '00), Dallas/Richardson, Texas, USA, pp. 232, Dec 2000.

[7] A. Nasipuri, J. Zhuang and S. R. Das, "A Multichannel CSMA MAC Protocol for Multihop Wireless Networks," Proceedings of IEEE Wireless Communications and Networking Conference (WCNC), Sep 1999.

[8] P. Porwal and M. Papadopouli, "On-Demand Channel Switching for Multi-Channel Wireless MAC Protocols," Technical Report TR04-024, 2004.

[9] J. So and N. H. Vaidya, "A Multi-Channel MAC Protocol for Ad Hoc Wireless Networks," Proceedings of ACM Mobihoc'04, May 2004.

[10] IEEE WG, IEEE 802.1lp/D2.01, Draft Amendement to Part 11: Wireless Medium Access Control (MAC) and Physical Layer (PHY) specifications: Wireless Access in Vehicular Environments, Mar 2007.

[11] http://www.itsa.org/

[12] S. Biswas, R. Tatchikou and Francois Dion, "Vehicleto-vehicle wireless communication protocols for enhancing highway traffic safety," IEEE Communications Magazine, pages 74-82, Jan 2006.

[13] H. W. So, J. Walrand, "Comparison of Multi-Channel MAC Protocols," Proceedings of 8th MSWiM, Montreal, Canada, Sep 2005.

[14] S. L. Wu , C. Y Lin and Y. C. Tseng, "A New Multi-Channel MAC Protocol with On-Demand Channel Assignment for Multi-Hop Mobile Ad Hoc Networks," Proceedings of 2000 International Symposium on Parallel Architectures, Algorithms and Networks (ISPAN '00), p. 232237, Dec 2000

[15] Berkeley Highway Lab (BHL), http://bhl.calccit.org/.

[16] B. Cash, "North American 5.9 GHz DSRC Operational Concept / Band Plan," doc.: IEEE 802.11-03/0750r0

[17] Y. B. Ko, V. Shankarkumar, and N. H. Vaidya, "Medium access control protocols using directional antennas in ad hoc networks", Proceedings of IEEE INFOCOM' 2000, Mar 2000.

[18] R. R. Choudhury, X. Yang and R. Ramanathan, "Using Directional Antennas for Medium Access Control for Ad Hoc Networks," Proceedings of ACM MobiCom 2002, Atlanta, Georgia, USA, pp. 59-70, Sep 2002.

[19] A. Nasipuri, J. Mandava and H. Manchala, "On-demand routing using directional antennas in mobile ad hoc networks," Proceedings of IEEE ICCCN 2000, Oct 2000. 